\title{
Amiloidosis. Comunicación de 11 casos y revisión de la literatura
}

\author{
Carmen Luz Palma $\mathbf{R}^{1}$, Daniela Grünholz $\mathbf{G}^{\mathbf{1}}$, \\ G uido $\mathbf{O}$ sorio $\mathbf{S}$.
}

\section{Clinical features of patients with the pathological diagnosis of amyloidosis}

Background: Amyloidosis is characterized by the extracellular deposit of an insoluble fibrillar protein that leads to tissue atrophy and necrosis. Aim: To report the clinical features of cases of amyloidosis diagnosed in a public hospital in Santiago, Chile, from 2000 to 2004. Material and methods: Retrospective review of all pathology reports of biopsies obtained from 2000 to 2004. In all cases reported as "amyloidosis", the clinical features of such patients were obtained from their medical records. Results: The medical records of 11 patients with amyloidosis were obtained (aged 35 to 71 year old, seven females). Seven had a systemic and four a localized disease. Six patients had primary amyloidosis and in one, it was secondary to a disseminated tuberculosis. Five patients with the generalized disease consulted for anarsarca, three for weight loss and 2 for chronic diarrhea. Patients with localized disease consulted for tonsil enlargement, dysphonia and skin lesions. Five patients with generalized disease had renal involvement and five had cardiac involvement. Three patients had malabsorption. Conclusions: The most common presentation of systemic amyloidosis is anasarca and renal involvement is common (Rev Méd Chile 2005; 133: 655-61).

(Key Words: Amyloidosis; Kidney failure, chronic; Tuberculosis)

Recibido el 23 de agosto, 2004. Aceptado en versión corregida el 25 de abril, 2005.

Servicio Medicina, Hospital Barros Luco Trudeau. Santiago de Chile.

${ }^{1}$ Médico Becario de Medicina Interna, Universidad de Chile. Hospital Barros Luco Trudeau.

L a amiloidosis es un desorden de etiología no aclarada. Se caracteriza por el depósito patológico extracelular de una sustancia fibrilar de origen proteico y estructura beta plegada insoluble (amiloide) en distintos tejidos, con el resultado de atrofia y necrosis, con pérdida de la estructura normal del tejido, lo que lleva a disfunción en mayor o menor grado según la cuantía del depósito ${ }^{1-8}$.

Correspondencia a: Dra. Carmen Luz Palma Robles. Miguel Claro 1691, Casa D. Teléfono 09-8705922. E mail: c_luzpalma@hotmail.com
Las fibrillas de amiloide son rígidas, no ramificadas, de 7 a $10 \mathrm{~mm}$ de ancho, de una longitud variable, insolubles y altamente resistentes a la acción enzimática ${ }^{9,13}$.

Es una enfermedad poco frecuente, con una incidencia anual de 8 casos por millón de habitantes y una prevalencia de 5 a 13 casos por millón de habitantes al año en Estados Unidos de Norteamérica (EE.UU) ${ }^{11}$.

En una revisión bibliográfica realizada desde 1939 a la fecha, se precisa que en Chile no se han publicado estudios de incidencia ni prevalencia. Sólo se encuentran publicados dos artículos en la Revista Médica de Chile, que consisten en revisión 
anátomo-clínica de 35 y 32 casos, en 1960 y 1961, respectivamente ${ }^{1,9}$. El resto de las publicaciones corresponden a casos clínicos aislados ${ }^{11,12,14,15}$.

El presente trabajo pretende evaluar el número de casos diagnosticados en el Hospital Barros Luco Trudeau y describir las formas clínicas de presentación de los mismos, y compararlos con lo comunicado en la literatura.

\section{MATERIAL Y MÉTODO}

Se trata de un estudio retrospectivo y descriptivo. Se revisaron todos los informes de biopsia desde enero de 2000 a abril de 2004 del Departamento de Anatomía Patológica del Hospital Barros LucoTrudeau, que correspondieron a 1.500 informes y las fichas de pacientes en control en Departamento de Hematología (400 fichas), del mismo centro en el mismo período. En los casos diagnosticados como amiloidosis, se recolectó mediante pauta la siguiente información: sexo, edad al momento del diagnóstico, motivo de la primera consulta, hallazgos al examen físico y pruebas de laboratorio.

Se debe mencionar que todos los casos tienen el diagnóstico realizado por estudio histológico. Sin embargo, en 2 pacientes el diagnóstico se realizó post-mortem. Tanto en las biopsias como en el estudio necrópsico, el amiloide fue reconocido por tinción con rojo Congo.

\section{RESULTADOS}

Se logró localizar y revisar las fichas de 11 pacientes con el diagnóstico de amiloidosis del total de fichas revisadas, tanto del Departamento de Anatomía Patológica como del Departamento de Hematología. Sus características anátomo-clínicas aparecen en Tabla 1.

En 2 de ellos el diagnóstico se realizó por necropsia. La amiloidosis puede clasificarse en

Tabla 1 Características de los 11 casos

\begin{tabular}{|c|c|c|c|c|c|}
\hline Caso & Sexo & Edad & $\begin{array}{l}\text { Tipo de } \\
\text { amiloidosis }\end{array}$ & Motivo de consulta & $\begin{array}{l}\text { Sitio anatómico } \\
\text { biopsiado }\end{array}$ \\
\hline 1 & F & 56 & Sistémica & $\begin{array}{l}\text { Baja de peso } \\
\text { diarrea crónica }\end{array}$ & Necropsia \\
\hline 2 & F & 67 & Sistémica & $\begin{array}{l}\text { Edema } \\
\text { síndrome nefrótico } \\
\text { baja de peso }\end{array}$ & $\begin{array}{l}\text { Mucosa gingival } \\
\text { renal } \\
\text { rectal }\end{array}$ \\
\hline 3 & F & 35 & Sistémica & $\begin{array}{l}\text { Edema } \\
\text { diarrea crónica } \\
\text { síndrome nefrótico } \\
\text { baja de peso }\end{array}$ & $\begin{array}{l}\text { Renal } \\
\text { intestino delgado }\end{array}$ \\
\hline 4 & F & 71 & Sistémica & $\begin{array}{l}\text { Síndrome nefrótico } \\
\text { edema }\end{array}$ & $\begin{array}{l}\text { Adenopatía } \\
\text { inguinal }\end{array}$ \\
\hline 5 & M & 71 & Sistémica & $\begin{array}{l}\text { Edema } \\
\text { síndrome nefrótico }\end{array}$ & $\begin{array}{l}\text { Renal } \\
\text { médula ósea }\end{array}$ \\
\hline 6 & M & 57 & Sistémica & Edema & Necropsia \\
\hline 7 & $\mathrm{~F}$ & 40 & Sistémica & Fractura patológica & Tejido óseo \\
\hline 8 & F & 50 & Localizada & Disfonía & Laringe \\
\hline 9 & M & 68 & Localizada & Lesión cutánea & Piel \\
\hline 10 & M & 64 & Localizada & $\begin{array}{l}\text { Disfonía } \\
\text { aumento de volumen } \\
\text { amigdalino }\end{array}$ & Amígdala \\
\hline 11 & F & 64 & Localizada & $\begin{array}{l}\text { Prurito y eritema } \\
\text { facial }\end{array}$ & Piel \\
\hline
\end{tabular}




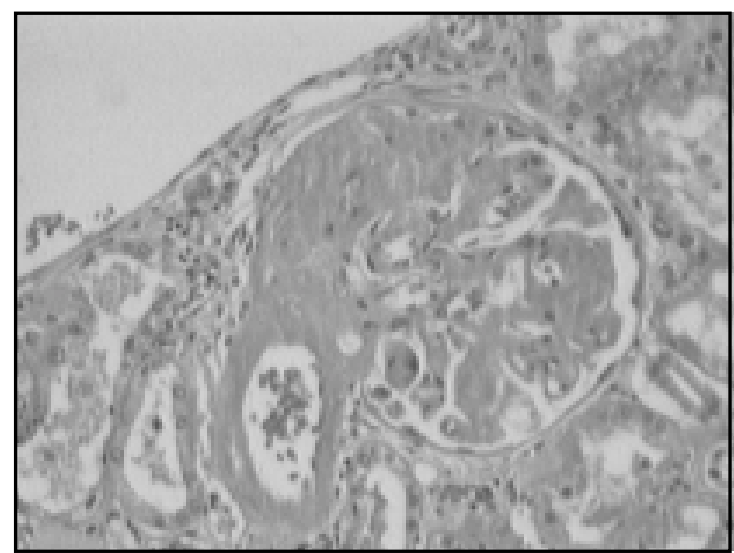

FIGURA 1. Compromiso renal en amiloidosis. Visión al microscopio óptico 40x tinción con hematoxicilinaeosina. Gentileza de Dr. Juan Rojas. Servicio de Anatomía Patológica, Hospital Barros-Luco Trudeau.

generalizada o sistémica y forma localizada, que corresponde al compromiso de un órgano sin signos de enfermedad generalizada.

Siete pacientes presentaron la enfermedad en forma sistémica, mientras que cuatro fueron casos de amiloidois localizada. El promedio de edad de los casos fue de 57,5 años (rango entre 35 y 71 años). La distribución por sexo reveló que 7 pacientes eran de sexo femenino y 4 de sexo masculino. En la serie hubo 7 casos de amiloidosis sistémica, 6 de amiloidosis primaria y un caso secundario a tuberculosis miliar. Con respecto a los 6 casos de amiloidosis primaria, hubo 2 pacientes que presentaron mieloma múltiple asociado.

En relación a los motivos de consulta, en los pacientes con amiloidosis generalizada, 5 que consultaron por edema y anasarca, 3 lo hicieron por baja de peso y compromiso del estado general y 2 casos se manifestaron clínicamente por síndrome diarreico crónico.

Los pacientes portadores de amiloidosis localizada consultaron por aumento de volumen amigdaliano en un caso, disfonía en otro y se reportaron lesiones cutáneas en 2 casos.

Con respecto al diagnóstico, se debe señalar que se realizó con estudio biópsico en 9 casos y en 2 pacientes se realizó por necropsia. En algunos de los casos se tiene estudio histológico de más de un sitio anatómico (Tabla 1).

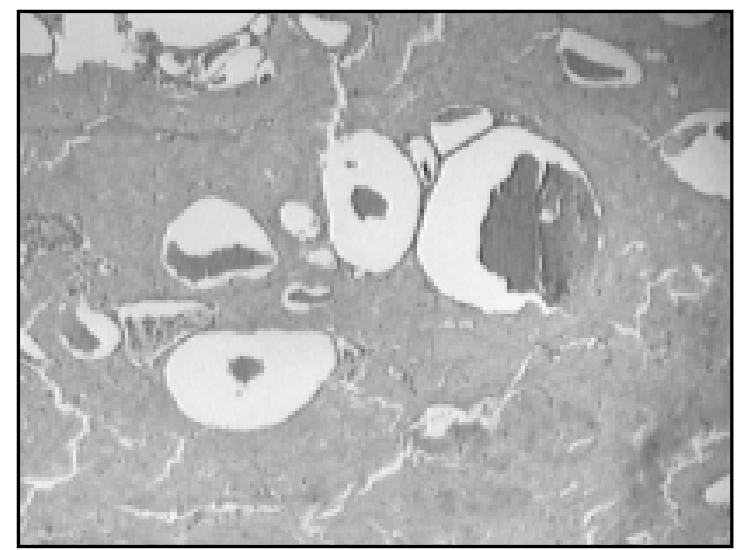

FIguRA 2. Tejido tiroideo infiltrado por sustancia amiloide. Visión al microscopio óptico 40x tinción con hematoxilina-eosina. Gentileza de Dr. Juan Rojas. Servicio de Anatomía Patológica, Hospital BarrosLuco Trudeau.

En relación al estudio realizado durante la hospitalización, se encontró que 5 de 7 pacientes con amiloidosis sistémica presentaron algún grado de compromiso renal, caracterizado por síndrome nefrótico y en tres de ellos con clearance de creatinina bajo $20 \mathrm{ml} / \mathrm{min}$, se requirió hemodiálisis durante la hospitalización. El compromiso histológico renal de uno de los casos se puede observar en la Figura 1 con tinción hematoxilina eosina.

Hubo 5 pacientes que presentaron compromiso cardíaco, evidenciado por ecocardiografía 2D en 3 casos, que se caracterizó por hipertrofia ventricular, dilatación de cavidades y por estudio necrópsico en los 2 casos restantes. En un paciente cuyo motivo de consulta fue dolor óseo, su diagnóstico definitivo fue de plasmocitoma de fémur izquierdo.

El síntoma digestivo más frecuente en esta serie fue la diarrea crónica, llegando a provocar en 3 casos síndrome de malabsorción y desnutrición calórico-proteica severa. Se evidenció compromiso tiroideo en 3 casos, caracterizado por bocio, hipotiroidismo e infiltración tiroidea en estudio necrópsico (Figura 2). En ninguno de los casos analizados se consignó en la ficha clínica la presencia de macroglosia, púrpura periorbitario ni ruptura esplénica. Tampoco los pacientes tenían antecedentes familiares de amiloidosis. Como dato anexo, 2 pacientes tenían asociación con enfermedad neoplásica, un caso de cáncer de ovario 
Tabla 2. H allazgos al laboratorio de pacientes con amiloidosis sistémica

\begin{tabular}{|ccccccc|}
\hline & Sexo & Edad & Creatinina & BUN & Ca & P 24 \\
\hline 1 & $\mathrm{~F}$ & 56 & 2,2 & 28 & 10 & 3,8 \\
2 & $\mathrm{~F}$ & 67 & 0,6 & 12 & 8 & 10,4 \\
3 & $\mathrm{~F}$ & 35 & 0,63 & 20 & 6 & 3,7 \\
4 & $\mathrm{~F}$ & 71 & 2,1 & 30 & 8 & 7,2 \\
5 & $\mathrm{M}$ & 71 & 1,29 & 20 & 9 & 6,2 \\
6 & $\mathrm{M}$ & 57 & 3,06 & 80 & 8 & 6,9 \\
7 & $\mathrm{~F}$ & 40 & 0,79 & 21 & 10 & 1 \\
\hline
\end{tabular}

P24: proteinuria de $24 \mathrm{~h}$ en gr/ $24 \mathrm{~h}$. Ca: Calcio en mg/dl. Creatinina en $\mathrm{mg} / \mathrm{dl}$.

Tabla 3. Electroforesis en 3 casos

\begin{tabular}{|c|c|c|c|}
\hline Caso & EFP en suero & $\begin{array}{l}\text { Tipo de Ig } \\
\text { principal }\end{array}$ & Cadena \\
\hline 1 & $\begin{array}{l}\text { Peak } \\
\text { monoclonal en } \\
\text { Gamma } 2\end{array}$ & $\operatorname{Ig} \mathrm{M}$ & Lambda \\
\hline 2 & $\begin{array}{l}\text { Peak } \\
\text { monoclonal en } \\
\text { Gamma 1 }\end{array}$ & Ig A & Lambda \\
\hline $3^{*}$ & $\begin{array}{l}\text { Peak } \\
\text { monoclonal en } \\
\text { Gamma } 3\end{array}$ & $\operatorname{IgG}$ & Lambda \\
\hline
\end{tabular}

EFP: electroforesis de proteínas. Ig: Inmunoglobulinas. *Caso asociado a mieloma múltiple.

Tabla 4. Clasificación de amiloidosis

\begin{tabular}{|ll|}
\hline Tipo & Nombre clínico \\
\hline Inmunoglobulina AL & Primaria \\
& asociada a mieloma múltiple \\
Reactiva AA & Secundaria \\
Hereditaria & Familiar \\
& Heredofamiliar \\
& Polineuropatía \\
B2 microglobulina & Diálisis \\
\hline
\end{tabular}

operado antiguo y un paciente cuya causa de muerte fue una hemorragia digestiva masiva secundaria a cáncer gástrico avanzado.

En la Tabla 2 se exponen algunos de los hallazgos de laboratorio general en los pacientes con amiloidosis sistémica. Sólo es posible presentar el estudio de inmunoelectroforesis de 3 pacientes que se detallan en la Tabla 3. No fue posible el financiamiento de la inmunohistoquímica en todos los casos vivos al momento del diagnóstico.

\section{DisCUSIÓN}

La historia de la amiloidosis se remonta al siglo XIX, cuando Virchow adoptó el término botánico amiloidosis para describir un material de depósito extracelular anormal encontrado en autopsias hepáticas. Posteriormente se detectó que dicho material, al teñirlo con rojo Congo, adquiría una coloración rojiza a la microscopia convencional y un tono verde manzana con luz polarizada. La naturaleza fibrilar del material se evidenció a través de la microscopia electrónica.

La amiloidosis puede clasificarse en términos generales en: amiloidosis primaria: sin signos de enfermedad coexistente, excepto mieloma múltiple; amiloidosis secundaria, con coexistencia de enfermedades inflamatorias o supurativas crónicas. Amiloidosis localizada: que corresponde al compromiso de un órgano sin signos de enfermedad generalizada. Familiar o hereditaria: muy poco frecuente y asociada a una mutación genética de la proteína transtirretina. Amiloidosis senil: que se encuentra como hallazgo localizado en autopsias aparentemente sin significado clínico. Amiloidosis de los hemodializados: en la que el depósito de amiloide está constituido por ß2microglobulina.

Amiloidosis primaria o AL: corresponde a $85 \%$ de los casos de las amiloidosis sistémica en EE.UU. Sus fibrillas son cadenas monoclonales livianas (lambda o kappa). Existe un tipo hereditario causado por mutaciones en diversos genes, extremadamente poco frecuente y que la mayoría de las veces no se incorpora como diagnóstico diferencial ${ }^{4}$. 
Amiloidosis secundaria o AA: en EE.UU y Europa es, generalmente, secundaria a artritis reumatoidea o enfermedad inflamatoria intestinal, pero en países subdesarrollados es más frecuente secundaria a tuberculosis o lepra. En este tipo de amiloidosis, las fibrillas son de proteína $\mathrm{A}^{7}$.

Las manifestaciones clínicas de la amiloidosis son diversas, variadas y dependerán de la distribución e intensidad de los depósitos de amiloide.

La amiloidosis AL representa la variedad más agresiva de amiloidosis sistémica, afectando típicamente riñón, aparato digestivo, hígado, suprarrenales, corazón, articulaciones y sistema nervioso central y periférico.

La afección renal, en el contexto de las amiloidosis sistémicas AA y AL, es prácticamente constante (90\%), siendo el compromiso glomerular el más frecuente. Cursa con proteinuria intensa, no selectiva y desarrollo de síndrome nefrótico en fases más evolucionadas 3,11,16.

El compromiso cardíaco es frecuente en el contexto de la amiloidosis sistémica, especialmente en la amiloidosis AL La infiltración del miocardio por sustancia amiloide provoca una alteración funcional en forma de insuficiencia cardíaca congestiva, cardiomegalia, arritmias, con intensa depresión de la función sistólica y diastólica, definiendo un patrón típico de cardiomiopatía restrictiva. La infiltración cardíaca representa el factor pronóstico más importante en la amiloidosis sistémica. Estudios recientes informan que podrían utilizarse troponina $\mathrm{T}$ e I como marcadores y predictores de sobrevida ${ }^{17}$. Así como la captación miocárdica de pirofosfato ${ }^{7}$.

La afección gastrointestinal es frecuente, la macroglosia es de las alteraciones más características y dificulta la masticación y deglución. Con frecuencia se observan cuadros de malabsorción, diarrea y hemorragia digestiva. La infiltración hepática por sustancia amiloide es frecuente en las formas sistémicas y se traduce como un síndrome colestásico anictérico y hepatomegalia homogénea ${ }^{18}$.

La afección neurológica se presenta especialmente en las formas hereditarias, en forma de polineuropatía mixta con afección autonómica. Esta última se manifiesta por hipotensión ortostática, incontinencia de esfínteres y alteraciones de la sudoración. Se describen además formas familia- res poco frecuentes ${ }^{15}$. El síndrome del túnel carpiano se puede observar en la amiloidosis AL y en la del paciente en hemodiálisis ${ }^{19}$.

La infiltración por sustancia amiloide de glándulas endocrinas cursa en general de forma asintomática, las glándulas tiroides y suprarrenal son las más frecuentemente afectadas ${ }^{12}$.

Para la realización del diagnóstico de amiloidosis se requiere un alto índice de sospecha. El diagnóstico definitivo se basa en la demostración en el estudio histológico del órgano afectado de los depósitos de sustancia amiloide que se identifican por sus características tintoriales específicas. El estudio debe incluir inmunohistoquímica con anticuerpos monoclonales que permitan realizar el diagnóstico específico de variedad de amiloido$\operatorname{sis}^{20}$.

La biopsia renal o hepática es positiva sobre 90\% de los casos. Sin embargo, se han publicado métodos menos invasivos, aunque con menor sensibilidad: punción aspirativa de grasa abdominal (60 a $80 \%)$, biopsia rectal (50 a 70\%), biopsia de médula ósea (50-55\%), entre otras ${ }^{23-26}$.

No existe un tratamiento curativo para la enfermedad. Se han usado tratamientos con quimioterapia, cuyos resultados hasta ahora han sido mínimos $^{2}$. El tratamiento estándar actual es una combinación de melfalán y prednisona, que destruiría a las células plasmáticas responsables de la producción de cadenas livianas ${ }^{10}$. Sin embargo, sólo $30 \%$ de los pacientes responde ${ }^{1,5}$. En el último tiempo, se están realizando estudios en algunos pacientes con trasplante de células troncales y quimioterapia, en pacientes en estadios tempranos de la enfermedad, con resultados prometedores. Los casos de amiloidosis secundaria, deben recibir tratamiento para su enfermedad de base ${ }^{2,8}$.

El diagnóstico precoz de amiloidosis requiere alta sospecha clínica, debido a que es una enfermedad poco frecuente y poco considerada, esto queda de manifiesto en los casos analizados, destacando que el diagnóstico definitivo fue realizado en 2 de los pacientes con estudio necrópsico.

Del análisis de los datos, se puede concluir que de los pacientes con amiloidosis sistémica, la mayoría tuvo compromiso renal, caracterizado por síndrome nefrótico, siendo el motivo de consulta 
más frecuente en esta serie el edema con mayor o menor grado de proteinuria, lo que coincide con la literatura $1,3,6,7,16,21$. Se debe mencionar que la enfermedad renal por depósitos de cadenas puede manifestarse de diversas formas, por lo que, a pesar de ser el síndrome nefrótico la manifestación más frecuente, se debe recordar que también se describen lesiones túbulo-intersticiales, vasculares y proteinuria de Bence-Jones asintomática ${ }^{16}$.

Al revisar los datos en relación a amiloidosis localizada no se sospechó el diagnóstico previo al estudio histológico y la hipótesis diagnóstica, en todos los casos, fue neoplasia maligna, debido a que el hallazgo o motivo de consulta más frecuente fue el aumento de volumen del órgano comprometido.

$\mathrm{Al}$ analizar y comparar con las series de casos clínicos publicados en Chile ${ }^{1,9,22}$, se debe mencionar el cambio en relación al perfil de la enfermedad. Es así como en las dos series anteriormente publicadas, destaca en ellas que el diagnóstico se realizó por necropsia en más de $90 \%$ de los casos, a diferencia de nuestra serie en que el diagnóstico se realizó por estudio biópsico. Otro punto a resaltar, corresponde a los tipos de amiloidosis encontrados en cada serie. En nuestra serie fueron principalmente casos de amiloidosis primaria, a diferencia de lo reportado en las otras dos series nacionales, en que la mayoría de los casos correspondió a amiloidosis secundaria, principalmente tuberculosis. En relación al motivo de

\section{REFERENCIAS}

1. Alvo M, Vera M, Melfi M. Amiloidosis renal. Estudio anátomo clínico de 32 casos. Rev Méd Chile 1960; 89: 181-6.

2. Skinner M, Sanchorawala V, Seldin D, Dember L, RODNEY H, BeRK L. High-dose melphalan and autologous stem-cell transplantation in patients with AL amyloidosis: An 8 years study. Ann Intern Med 2004; 140: 85-93.

3. Kyle R. Monoclonal Proteins and Renal Disease. Annu Rev Med 1994; 45: 71-7.

4. Lachmann H, Booth D, Chir B, Booth S, Bybee A, GiLBERTSON J. Misdiagnosis of hereditary amyloidosis as AL (primary) amyloidosis. N Engl J Med 2002; 346: 1786-91. consulta, en ambas series correspondió a edema y compromiso renal. Ello concuerda además con la literatura extranjera. El compromiso gastrointestinal caracterizado por diarrea crónica y demostrado además por estudio histológico fue frecuente tanto en las series revisadas así como en la nuestra. Con respecto a la edad de presentación, se puede observar que en nuestra serie tuvo promedio de 57 años, a diferencia de lo reportado en las series ya mencionadas, en la edad fue menor (40 años en promedio).

Se debe mencionar que sólo en tres pacientes se practicaron estudios de inmuhistoquímica, la cadena liviana presente en todos los casos correspondió a la cadena lambda, como se observa en la Tabla 3.

En conclusión el presente trabajo describe las formas clínicas de presentación de los casos de amiloidosis diagnosticados en el Hospital Barros Luco Trudeau, desde 2000. El compromiso renal fue el hallazgo más frecuente lo que coincide con la literatura tanto nacional como extranjera.

En los casos diagnosticados en nuestro hospital, predominan las formas de amiloidosis primaria y el diagnóstico se realizó, la mayoría de las veces, por estudio biópsico, a diferencia de lo que muestran las series publicadas anteriormente en Chile, en que la mayoría de los casos correspondieron a formas secundarias a tuberculosis, y en las cuales el diagnóstico fue realizado por necropsia en casi la totalidad de los casos.

5. Marín P, Fardelia C, Rosenberg H. Nuevo método para el diagnóstico de amiloidosis: Biopsia por punción del tejido subcutáneo. Rev Méd Chile 1987; 115: 760-2.

6. Moroni A, Benavides A, Retamal Y. Macroglosia y amiloidosis oculta. Rev Méd Chile 2002; 130: 209-14.

7. Redondo F, González P, Ramírez A. Captación miocárdica de pirofosfato en amiloidosis cardíaca. Caso clínico. Rev Méd Chile 2002; 130: 315-8.

8. DisPENZIERI A. Amyloid proteins in pathogenesis of AA amyloidosis. Lancet 2001; 358: 24-9.

9. Gazmuri $\mathrm{O}$, UbiLi V, Vera M, Arís H. Amiloidosis. Revisión anátomo-clínica de 35 casos. Rev Méd Chile 1961; 88: 44-8.

10. Gertz M, Lacy M, Lust J, GreipP P, Witzig T, Kyle R. Prospective randomized trial of melphalan and 
prednisone versus vincristine, carmustine, melphalan cyclophosphamide and prednisone in the treatment of primary systemic amyloidosis. J Clin Oncol 1999; 17: 262-7.

11. Lulo M, Dupommereu山e C, Prieur P, Petrover M. Depósito de sustancia amiloide y niveles plasmáticos de B2 microglobulina en pacientes en hemodiálisis crónica. Rev Méd Chile 1987; 5: 486-7.

12. Télezz R, Le Cerf P, Araos F, Michaud P. Infiltración adiposa difusa de la glándula tiroides asociada a amiloidosis en un paciente con insuficiencia renal crónica. Rev Méd Chile 1996; 5: 1951-6.

13. MuÑoz J. Diagnóstico y seguimiento de la amiloidosis. Rev Esp Reumatol 1996; 23: 21-5.

14. Gutiérrez M, Torrealba B, Mendoza J, Naquira N. Amiloidosis sistémica primaria con compromiso pulmonar difuso. Causa infrecuente de enfermedad intersticial. Rev Méd Chile 1997; 10: 1187-92.

15. Guevara C, Barrientos N, Flores A, idiáouez J. Polineuropatía amiloidótica familiar tipo I. Rev Méd Chile 2003; 131: 1179-82.

16. SANDERS P, HeRrera G. Monoclonal inmunoglobulin light chain, related renal diseases. Semin Nephrol 1993; 13: 324-41.

17. Dispenzieri A, Kyle R, Gertz M, Therneau T, Milar W, Chandrasekaran K. Survival in patients with primary systemic amyloidosis and raised serum cardiac troponins. Lancet 2003; 361: 1787-9.

18. Berríos M, Armas-Merino R, Franco C, Parrochia E, Wolf C. Insuficiencia hepática aguda en amiloidosis hepática asociada a mieloma múltiple. Caso clínico. Rev Méd Chile 2003; 131: 1301-4.
19. FerRer S, JimÉnez P. Polineuropatías y disglobulinemias. Rev Méd Chile 1994; 122: 448-53.

20. BuxBAum J. Mechanisms of disease: monoclonal immunoglobulin deposition. Hematol Oncol Clin of North Am 1992; 6: 323-46.

21. Buxmaum J, Chuba J, Helman G, Solomón A, Galo G. Monoclonal inmunoglobulin deposition disease: Light chain and light and heavy chain deposition disease and their relation to light chain amyloidosis. Am Intern Med 1990; 112: 455-64.

22. Solar E, Olivares O, Urrutia S. Amiloidosis rinofaringo-laringotraqueal. Caso clínico. Rev Méd Chile 1967; 95: 93-5.

23. Ansari-Lari MA, Ali SZ. Fine-needle aspiration of abdominal fat pad for amyloid detection: a clinically useful test? Diagn Cytopathol 2004; 30: 178-81.

24. Masouye I. Diagnostic screening of systemic amyloidosis by abdominal fat aspiration: an analysis of 100 cases. Am J Dermatopathol 1997; 19: 41-5.

25. Arbustini E, Verga L, Concardi M, PaLadini G, Obici L, MERLINI G. Electron and immuno-electron microscopy of abdominal fat identifies and characterizes amyloid fibrils in suspected cardiac amyloidosis. Amyloid 2002; 9: 108-14.

26. GuY CD, JoNES CK. Abdominal fat pad aspiration biopsy for tissue confirmation of systemic amyloidosis: specificity, positive predictive value, and diagnostic pitfalls. Diagn Cytopathol 2001; 24: 181-5.

Agradecimientos

Los autores agradecen, de forma especial, al Dr. Juan Luis Rojas Pavez del Departamento de Anatomía Patológica del Hospital Barros Luco Trudeau, por su aporte de material fotográfico e histológico. 